\title{
DiGeorge subtypes of nonsyndromic conotruncal defects: evidence against a major role of $T B X 1$ Gene
}

\author{
Emanuela Conti $^{1}$, Nicoletta Grifone ${ }^{1}$, Anna Sarkozy ${ }^{1,2}$, Caterina Tandoi ${ }^{1,2}$, Bruno Marino ${ }^{3}$, \\ Maria Cristina Digilio ${ }^{4}$, Rita Mingarelli ${ }^{1}$, Antonio Pizzuti ${ }^{1,2}$ and Bruno Dallapiccola ${ }^{1,2}$
}

${ }^{1}$ CSS Hospital, IRCCS, San Giovanni Rotondo, Italy, and CSS-Mendel Institute, Rome, Italy; ${ }^{2}$ Section of Medical Genetics, Department of Experimental Medicine and Pathology, University 'La Sapienza', Rome, Italy; ${ }^{3}$ Section of Pediatric Cardiology, Institute of Pediatrics, University 'La Sapienza', Rome, Italy; ${ }^{4}$ Division of Medical Genetics, Bambino Gesù Hospital, IRCCS, Rome, Italy

The role of the $22 q 11$ region genes, and among them $T B X 1$, in nonsyndromic conotruncal defects (CTDs) is still unclear. Mice hemizygous at the $T b \times 1$ locus show a remarkable incidence of heart outflow tract anomalies, of the same type commonly found in DiGeorge/Velo-cardio-facial syndrome (DGS/VCFS). Mutation analysis of the TBX1 gene in isolated, nonsyndromic CTDs has not demonstrated any functional pathogenetic variation so far. We screened the $T B X 1$ gene in 41 patients affected by nonsyndromic CTDs of the DGS/VCFS subtype, principally 'atypical' tetralogy of Fallot. Besides a few polymorphisms, we did not find any pathogenetic variation. These results do not support a major role of the TBX1 gene as responsible for human nonsyndromic CTDs.

European Journal of Human Genetics (2003) 11, 349-351. doi:10.1038/sj.ejhg.5200956

Keywords: TBX1; conotruncal heart defects; DiGeorge/Velo-cardio-facial syndrome

\section{Introduction}

Conotruncal defects (CTDs) represent an important class of congenital heart diseases. Although they are mostly considered as complex, multifactorial disorders, ${ }^{1}$ a number of familial cases suggesting Mendelian inheritance have been described. ${ }^{2,3}$ CTDs, primarily interrupted aortic arch, persistent truncus arteriosus and tetralogy of Fallot (ToF), often occur in DiGeorge/Velo-cardio-facial syndrome (DGS/VCFS) patients with chromosome 22q11.2 deletion (del22q) ${ }^{4}$ However, del22q is very rare in isolated CTDs. In a cohort of 628 CTD patients we found only a single case of del22q. ${ }^{5}$ Even if recent studies have reported nonsyndromic ToF with NKX2.5 and JAG1 gene mutations, ${ }^{3,6}$ singlegene defects are likely to account for a very minority of CTD cases. In addition, the exclusion of del22q does not

*Correspondence: $\operatorname{Dr}$ E Conti, CSS-Mendel Institute, Viale Regina Margherita 261, 00198 Rome, Italy. Tel: +39 6 44160503; Fax: +39 6 44160548; E-mail: e.conti@css-mendel.it

Received 6 August 2002; revised 13 December 2002; accepted 17 December 2002 modify the recurrence risk in isolated non-syndromic CTDs. ${ }^{7}$ All the evidences, therefore, agree with the multifactorial model of transmission for isolated CTDs and suggest that many genes and pathogenetic mechanisms may be at work.

The TBX1 gene maps to the commonly deleted DGS/ VCFS region on chromosome $22 \mathrm{q} 11.2$ and codes for a transcription factor belonging to the T-box family, ${ }^{8}$ probably required for growth and septation of the conotruncus. ${ }^{9}$ Mice hemizygous at the Tbx1 locus show a remarkable incidence of heart outflow tract anomalies, of the same type found in DGS/VCFS patients, ${ }^{10,11}$ suggesting a role of TBX1 in the pathogenesis of human CTDs. ${ }^{10-12}$ However, a few independent TBX1 gene mutation screenings in DGS/VCFS patients without del22q turn out negative. ${ }^{8,10}$ Gong et al ${ }^{13}$ have investigated TBX1 both in nondeleted patients with features of DGS/VCFS and in a series of subjects with isolated CTDs, including aortic arch anomalies, which can occur in DGS/VCFS individuals. They found a few rare TBX1 variants in four DGS/VCFS patients and in three isolated CTDs, but a functional role 
for these variants was not demonstrated. In order to better address the issue of the link between TBX1 and isolated CTDs, we screened TBX1 in a large sample of patients, including some peculiar anatomical CTD subtypes which were not represented in the mutation screening previously performed. ${ }^{13}$

\section{Materials and methods}

A total of 41 Italian patients, ranging in age from birth to 16.5 years (mean age $\pm \mathrm{SD}=3.2 \pm 1.7$ years), were selected on strict phenotypic and cardiac criteria. Diagnoses were obtained by echocardiography and cardiac catheterization. In addition to interrupted aortic arch type $B,{ }^{14}$ other CTD subtypes typically associated with del22q were included in the study, such as 'atypical' ToF with absent pulmonary valve or pulmonary atresia and major aorto-pulmonary collateral arteries, ${ }^{15,16}$ pulmonary atresia with ventricular septal defects, ${ }^{17}$ and truncus arteriosus with truncal valve dysplasia ${ }^{18}$ (Table 1). Patients showed neither major or minor extracardiac features of DGS/VCFS, nor deletion of 22q11 and 10p13 DiGeorge syndrome regions by standard molecular analysis. ${ }^{19}$ Parental informed consent was obtained. The TBX1 gene coding regions including exon-intron boundaries and $500 \mathrm{bp}$ upstream the start codon were PCR amplified from genomic DNA. Primers were designed following the three splicing variant cDNA sequences and the corresponding genomic regions available on the GenBank database (AF012130, AF012131 and AF373867 and AC000091). The first eight exons are present in all the three differently spliced forms, while exon 9 and 10 primers had to be designed for each alternative cDNA (TBX1A, TBX1B and TBX1C) (Figure 1). PCR products were screened by single-strand conformation polymorphism analysis (SSCP; Genephor Unit; Amersham Pharmacia Biotech, Uppsala, Sweden). All fragments showing anomalous mobility shifts were sequenced (ABI PRISM 310 Genetic Analyser automated sequencer; Applied Biosystems, Foster City, CA, USA).

Table 1 Cardiac phenotype of the 41 screened patients

Congenital heart defects No. of patients

Tetralogy of Fallot
with pulmonary atresia and
major aorto-pulmonary collateral arteries
with absent pulmonary valve
with aorto-pulmonary window
Truncus arteriosus with truncal valve dysplasia
Interrupted aortic arch type B

Total

20

4

2

11

4

41

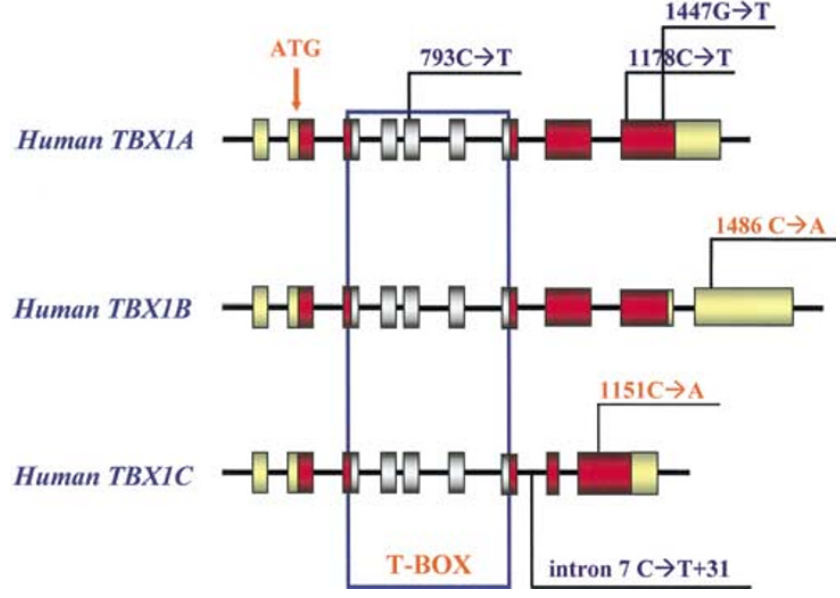

Figure 1 Genomic representation of the three alternative isoforms of the TBX1 gene (TBX1A, TBX1B and TBX1C) and position of the detected polymorphisms. Red boxes indicate coding exons and are drawn to scale, gray boxes, pointed out by the blue line, indicate T-box coding exons, shared by all three transcripts; yellow boxes represent nontranslated sequences ( $5^{\prime}$ and $3^{\prime}$ UTR). The intronic regions are not to scale. The start codon position is shown by the arrow. Newly detected polymorphisms are represented in red.

\section{Results}

Six heterozygous sequence variations were detected in exons 5, 7, 9A, 9C and 10 (Figure 1). The variations in exons $5(793 \mathrm{C} \rightarrow \mathrm{T})$ and $9 \mathrm{~A}(1178 \mathrm{C} \rightarrow \mathrm{T} ; 1447 \mathrm{G} \rightarrow \mathrm{T})$, as well as in intron $7(\mathrm{C} \rightarrow \mathrm{T}+31)$ had been previously reported and represent common polymorphisms. ${ }^{8,13}$ Analysis of exon 9 of the TBX1C mRNA form revealed a $\mathrm{C} \rightarrow \mathrm{A}$ substitution at nucleotide 1151 (Pro384Gln). Analysis of exon 10 of the TBX1B mRNA displayed a $\mathrm{C} \rightarrow \mathrm{A}$ substitution at position 1486 in the $3^{\prime}$ untranslated sequences. The Exon 9C variant was identified in two out of 100 nonaffected Italian controls with an allele frequency of $1 \%$, while the exon $10 \mathrm{~B}$ variant was present in 21 normal controls (allele frequency $10.5 \%$ ). No pathogenic mutation was identified.

\section{Discussion}

This study has analyzed the TBX1 gene including the $5^{\prime}$ and $3^{\prime}$ untranslated regions and the putative promoter for mutations in isolated CTD subtypes characteristically occurring in DGS/VCFS. The results provide independent confirmation that $T B X 1$ mutations are not a significant cause of isolated subtypes of conotruncal defects.

These findings, while agreeing with most of the previous studies on DGS/VCFS patients without 22q11 deletion, apparently contrast those observed by Gong et al, ${ }^{13}$ who reported rare $T B X 1$ variants in isolated aortic arch anomalies. All the TBX1 variants involved a newly discovered exon 9C, which was not screened in the previous negative studies. As remarked by Gong et al ${ }^{13}$ in 
their report, TBX1 mutations may be extremely rare, accounting only for a few CTD cases. Together with a quite different distribution of the CTD subtypes in our sample, it may explain the diverging results between the two studies.

The TBX1 gene variations so far reported on isolated CTDs are either polymorphisms, or changes with doubtful functional value. ${ }^{20}$ We cannot anyway rule out the possibility that some of these variants, even with wide distribution in the population, are somehow involved in the pathogenesis of the patients' heart malformations, interacting with other genetic and nongenetic factors, not strictly associated with the DGS/VCFS region, in accordance with the multifactorial model. Future functional studies will address this issue.

In conclusion, the present study does not support a major role of the TBX1 gene in the pathogenesis of human nonsyndromic CTDs. TBX1 sequence variations are not likely to represent causative factors of this type of human cardiac defects.

\section{Acknowledgements}

This study was supported in part by grants from the Italian Ministry of Health (RF 2002) and from the Italian Ministry of Instruction, University and Research (Grant $40 \%$ to BD).

\section{References}

1 Burn J, Brennan P, Little J et al: Recurrence risks in offspring of adults with major heart defects: results from first cohort of British collaborative study. Lancet 1998; 351: 311-316.

2 Pacileo G, Musewe NN, Calabro R: Tetralogy of Fallot in three siblings: a familial study and review of the literature. Eur J Pediatr 1992; 151: 726-727.

3 Eldadah ZA, Hamosh A, Biery NJ et al: Familial tetralogy of Fallot caused by mutation in the Jagged1 gene. Hum Mol Genet 2001; 10: $163-169$.

4 Marino B, Digilio MC, Toscano A, Giannotti A, Dallapiccola B: Congenital heart defects in patients with DiGeorge/ velocardiofacial syndrome and del22q11. Genet Couns 1999; 10: 25-33.

5 Marino B, Digilio MC, Toscano A et al: Anatomic patterns of conotruncal defects associated with deletion 22q11. Genet Med 2001; 3: 45-48.
6 Goldmuntz E, Geiger E, Benson DW: NKX2.5 mutations in patients with tetralogy of Fallot. Circulation 2001; 104: 2565-2568.

7 Digilio MC, Marino B, Giannotti A, Toscano A, Dallapiccola B: Recurrence risk figures for isolated tetralogy of Fallot after screening for 22q11 microdeletion. I Med Genet 1997; 34: 188-190.

8 Chieffo C, Garvey N, Gong W et al: Isolation and characterization of a gene from the DiGeorge chromosomal region homologous to the mouse Tbx1 gene. Genomics 1997; 43: 267-277.

9 Vitelli F, Morishima M, Taddei I, Lindsay EA, Baldini A: Tbx1 mutation causes multiple cardiovascular defects and disrupts neural crest and cranial nerve migratory pathways. Hum Mol Genet 2002; 11: 915-922.

10 Lindsay EA, Vitelli F, Su H, Morishima $M$ et al: Tbx1 haploinsufficiency in the DiGeorge syndrome region causes aortic arch defects in mice. Nature 2001; 410: 97-101.

11 Jerome LA, Papaioannou VE: DiGeorge syndrome phenotype in mice mutant for the T-box gene, Tbx1. Nat Genet 2001; 27: 286-291.

12 Merscher S, Funke B, Epstein JA et al: TBX1 is responsible for cardiovascular defects in velo-cardio-facial/DiGeorge syndrome. Cell 2001; 104: 619-629.

13 Gong W, Gottlieb S, Collins J et al: Mutation analysis of TBX1 in non-deleted patients with features of DGS/VCFS or isolated cardiovascular defects. J Med Genet 2001; 38: E45.

14 Momma K, Ando M, Matsuoka R, Joo K: Interruption of the aortic arch associated with deletion of chromosome 22q11 is associated with a subarterial and doubly committed ventricular septal defect in Japanese patients. Cardiol Young 1999; 9: 463-467.

15 Momma K, Kondo C, Ando M, Matsuoka R, Takao A: Tetralogy of Fallot associated with chromosome 22q11 deletion. Am J Cardiol 1995; 76: 618-621.

16 Marino B, Digilio MC, Grazioli S et al: Associated cardiac anomalies in isolated and syndromic patients with tetralogy of Fallot. Am J Cardiol 1996; 77: 505-508.

17 Digilio MC, Marino B, Grazioli S, Agostino D, Giannotti A, Dallapiccola B: Comparison of occurrence of genetic syndromes in ventricular septal defect with pulmonic stenosis (classic tetralogy of Fallot) versus ventricular septal defect with pulmonic atresia. Am J Cardiol 1996; 77: 1375-1376.

18 Marino B, Digilio MC, Dallapiccola B: Severe truncal valve dysplasia: association with DiGeorge syndrome. Ann Thorac Surg 1998; 65: 517-524.

19 Desmaze C, Scambler P, Prieur M et al: Routine diagnosis of DiGeorge syndrome by fluorescent in situ hybridization. Hum Genet 1993; 90: 663-665.

20 McDermid HE, Morrow BE: Genomic disorders on 22q11. Am J Hum Genet 2002; 70: 1077-1088. 